\title{
Use of wearable devices for post-discharge monitoring of ICU patients: a feasibility study
}

\author{
Ryan R. Kroll ${ }^{1 \dagger}$, Erica D. McKenzie ${ }^{2 \dagger}$, J. Gordon Boyd ${ }^{1,3}$, Prameet Sheth $^{4}$, Daniel Howes ${ }^{1,5}$, Michael Wood ${ }^{6}$, \\ David M. Maslove ${ }^{1,3,7^{*}}$ (D) and for the WEARable Information Technology for hospital INpatients (WEARIT-IN) study \\ group
}

\begin{abstract}
Background: Wearable devices generate signals detecting activity, sleep, and heart rate, all of which could enable detailed and near-continuous characterization of recovery following critical illness.

Methods: To determine the feasibility of using a wrist-worn personal fitness tracker among patients recovering from critical illness, we conducted a prospective observational study of a convenience sample of 50 stable ICU patients. We assessed device wearability, the extent of data capture, sensitivity and specificity for detecting heart rate excursions, and correlations with questionnaire-derived sleep quality measures.

Results: Wearable devices were worn over a 24-h period, with excellent capture of data. While specificity for the detection of tachycardia was high (98.8\%), sensitivity was low to moderate (69.5\%). There was a moderate correlation between wearable-derived sleep duration and questionnaire-derived sleep quality $(r=0.33, P=0.03)$. Devices were well-tolerated and demonstrated no degradation in quality of data acquisition over time.

Conclusions: We found that wearable devices could be worn by patients recovering from critical illness and could generate useful data for the majority of patients with little adverse effect. Further development and study are needed to better define and enhance the role of wearables in the monitoring of post-ICU recovery.
\end{abstract}

Trial registration: Clinicaltrials.gov, NCT02527408

Keywords: Wearable devices, Medical informatics, Mobile health technologies, Validation study, Critical care, Sleep quality, Heart rate monitoring

\section{Background}

Consumer interest in personal health tracking has recently increased, leading to an industry in wearable devices now valued at more than $\$ 9$ billion worldwide [1]. With more wearables in use than ever before, there has been growing enthusiasm for their potential to improve health care delivery [2]. Current clinical uses for wearable devices are mostly limited to outpatient settings, with a focus on the management of chronic diseases [3-5]. Newer generation

\footnotetext{
* Correspondence: david.maslove@queensu.ca

${ }^{\dagger}$ Equal contributors

'Department of Critical Care Medicine, Queen's University and Kingston Health Sciences Centre, Kingston, Ontario, Canada

${ }^{3}$ Department of Medicine, Queen's University and Kingston Health Sciences Centre, Kingston, Ontario, Canada

Full list of author information is available at the end of the article
}

devices generate data that could also be useful in characterizing convalescence from acute illness. These include photoplethysmography (PPG) sensors to detect heart rate $[6,7]$, as well as accelerometers to track activity and movement $[3,8,9]$.

Frequent heart rate tracking has the potential to identify episodes of clinical deterioration early. Accelerometer data could potentially be used to encourage mobilization, objectively measure functional status, and track progress towards rehabilitation goals. Wrist-worn accelerometers have also been used to evaluate sleep quality in healthy subjects $[10,11]$. In the inpatient and intensive care unit (ICU) settings, where poor sleep has been linked with adverse outcomes [12, 13], data describing sleep quality 
may be useful in identifying targets for sleep-promoting interventions [14].

There is little clinical evidence to inform the practice of using wearables in health care, most of which is focused on chronic conditions. Newer consumer-grade wearables have been evaluated in only a handful of studies examining their accuracy among healthy volunteers [3-5]. These studies have called for evaluations of this technology among a wider range of patient populations.

In this study, we examine the feasibility of using a common consumer-grade wearable device to monitor patients recovering from critical illness. We enrolled patients who no longer required intensive care measures but remained in the ICU prior to ward transfer, in order to best approximate post-ICU settings like the general wards, while still collecting gold standard data to validate device functionality. We report on a number of practical considerations that could affect the deployment of wearables including overall wearability, completeness of data capture, device longevity, and risk of transmitting nosocomial infections. We also evaluated the accuracy of wearables for measuring sleep quality and identifying changes in heart rate that might be clinically relevant. We hypothesized that patients recovering from critical illness would be able to wear wrist-worn devices and that useful data could be collected from these with a moderate degree of accuracy.

\section{Methods}

\section{Patients and setting}

This prospective observational study was conducted in a 33-bed general medical-surgical/trauma ICU in southeastern Ontario, between August 2015 and February 2016. Adult patients (age >17) were included if they were receiving continuous cardiac and oxygen saturation monitoring, but were otherwise receiving ward-level treatment. Exclusion criteria included mechanical ventilation, vasopressor support, and continuous sedation or analgesia. We specifically chose to study patients who were still in the ICU, as this was the most practical way to obtain gold standard measurements of heart rate and sleep quality, which would otherwise require the use of Holter monitors and complex follow-up procedures. To reduce the potential risk of transmitting nosocomial infections, patients under contact precautions for methicillinresistant Staphylococcus aureus (MRSA) and Clostridium difficile infections were also excluded. We also excluded patients at risk of vascular compromise of the arm on which the wearable device was to be placed, such as patients with upper extremity deep venous thrombosis, peripherally inserted central catheters, radial arterial lines, dialysis fistulas, and severe upper extremity trauma. As this was a feasibility study, a convenience sample of 50 participants was recruited.

\section{Ethics, consent, and permissions}

All participating patients, or substitute decision makers on their behalf, provided written informed consent for participation in this study. The Health Sciences Research Ethics Board at Queen's University reviewed and approved the study protocol (DMED-1818-15), and the trial was registered with clinicaltrials.gov (NCT02527408).

\section{Device}

Participating patients wore the Fitbit Charge HR device (Fitbit, San Francisco, CA, USA) for a single 24-h period (Fig. 1). The Fitbit Charge HR is a commercially available wrist-worn wearable that records heart rate, steps, and sleep quality. The study employed three size large wearable devices (15.7 to $19.3 \mathrm{~cm}$ wrist circumference) and three size extra-large wearable devices (19.6 to $22.6 \mathrm{~cm}$ wrist circumference). In an effort to reduce the risk of potential iatrogenic infection, we used disinfectant wipes to thoroughly clean wearables between uses. All devices were applied to participants by a study investigator or coordinator.

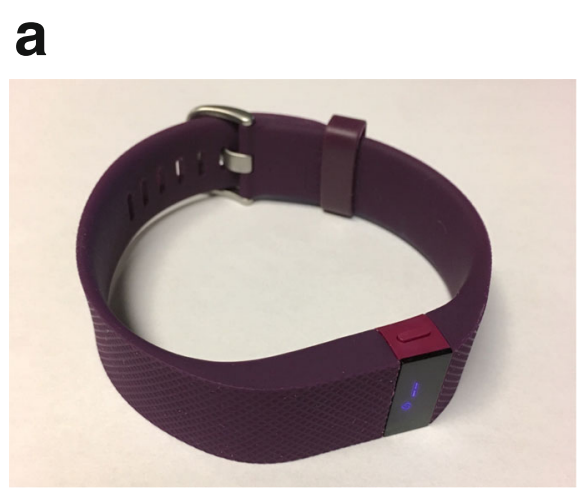

b

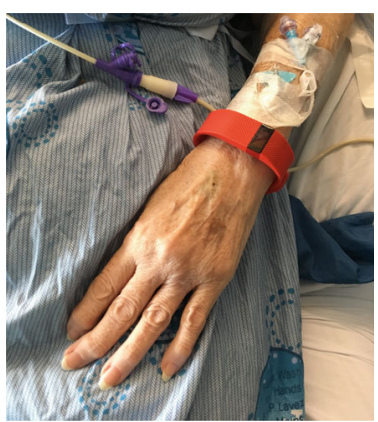

Fig. 1 The Fitbit Charge HR device used in the study (a). The wearable device as worn by a patient on the inpatient ward following ICU discharge (b) 


\section{Data monitoring and capture}

We used continuous pulse oximetry pulse rate recordings (SPO2-R) as a comparison measure of heart rate (HR) in order to evaluate the ability of wearables to detect both tachycardia (HR > $100 \mathrm{bpm}$ ) and bradycardia $(\mathrm{HR}<50 \mathrm{bpm})$. We used SPO2-R values as a comparator as both SPO2-R and wearable device values reflect the pulse rate (rather than electrical heart rate), and because this is a widely accepted method of heart rate measurement. The wearables recorded heart rate values every $5 \mathrm{~min}$, while the SPO2-R recorded heart rate values every minute. Cardiac rhythm was assessed at the time of device application, and again at the time of removal, at which time data regarding sleep quality was also collected using the Richards-Campbell Sleep Questionnaire (RCSQ) [15]. This survey uses a visual analog scale to assess sleep depth, latency, awakenings, percentage of time awake, and overall quality of sleep. The RCSQ was completed either by the patients themselves or by their designated night shift nurse, a practice previously shown to have slight to moderate agreement with self-assessment [16]. Due to the interaction between sleep and delirium in the ICU [17], patients were screened for delirium by a trained researcher using the confusion assessment method (CAM)-ICU at the time of device application, and again at the time of device removal.

Wearable-reported sleep data included time of sleep onset and awakening, sleep duration, minutes asleep, minutes awake, restless count, and a calculated measure of sleep quality. Overall sleep quality was taken as the average across sleep episodes, weighted by the duration of each sleep episode. The percentage of total sleep occurring during nighttime hours, which we defined as 22:00 to 06:00, and the percentage of nighttime hours spent asleep were calculated. For participants who had no Fitbitdetected sleep over the recording period, a score of 0 was given for all sleep parameters. Methods for obtaining wearable and SPO2-R data are reported elsewhere [18], and in the Supplementary Content (see Additional file 1).

\section{Microbiological assessment}

We conducted microbiologic sampling of the wearables used from a convenience subset of patients $(n=16)$ in order to evaluate both the risk of transmitting nosocomial pathogens from repeated application of wearables to different patients, as well as the efficacy of our disinfection practices (see Additional file 1).

\section{Statistical analysis}

In the absence of preliminary data to inform a sample size calculation, we targeted an enrollment of 50 patients, a cohort size equal to that used in a similar study of wrist-worn wearables for heart rate tracking in healthy volunteers [5]. In addition to basic descriptive statistics, we calculated the sensitivity and specificity of the wearables for detecting tachycardia and bradycardia. Based on the PPG mechanism of heart rate sensing employed in consumer-grade wearables, we hypothesized that the accuracy of wearable device heart rate tracking may be different in patients not in sinus rhythm and further analyzed these patients as a subgroup. We calculated Pearson correlation coefficients between the various wearable-derived measures of sleep quality and the RCSQ measures of sleep quality. Based on the mechanism of sleep sensing, which relies on the absence of movement, we hypothesized that the accuracy of wearables for sleep tracking may differ in patients with delirium, and further analyzed these patients as a subgroup. Statistical analyses for this study were performed using $\mathrm{R}$ (v 3.2.2).

\section{Results}

\section{Patients and device wearability}

We enrolled a total of 50 patients between August 2015 and January 2016 (Table 1). The median wrist circumference in our cohort was $18.6 \mathrm{~cm}$ (SD $1.9 \mathrm{~cm})$, with 6 of the 50 patients enrolled having moderate or severe edema of the wrist at the time of device application. The size large device was used for 23 patients (46\%), while the size extra-large was used for 27 patients (54\%). While there were no patients for whom the wearable device could not be fitted, the fit was noted to be very tight

Table 1 Characteristics of patients included in the study $(n=50)$

\begin{tabular}{lll}
\hline Mean heart rate (bpm) & 88.3 & \\
\cline { 2 - 3 } Mean age (years) & 64 & 52 \\
\cline { 2 - 3 } Patients $(n=50)$ & 48 \\
\hline Female & 26 & \\
Admission diagnosis & 24 & 24 \\
Respiratory & 12 & 14 \\
Sepsis & 7 & 14 \\
Surgical & 7 & 22 \\
Neurologic & 11 & 6 \\
Trauma & 3 & 12 \\
Cardiovascular & 6 & 8 \\
Medical & 4 & 86 \\
Sinus rhythm & & 84 \\
At start of monitoring & 43 & \\
At end of monitoring & 42 & 54 \\
Personal fitness tracker size used & & \\
Large & 23 & \\
Extra large & 27 & \\
\hline
\end{tabular}


in one patient, and very loose in two patients. Devices were adjusted only once at the time of application and were not re-assessed by study personnel for the duration of the 24-h recording period. No intravenous lines were re-sited in order to facilitate application, although hospital identification wristbands had to be relocated in some cases. No wearables required removal during the monitoring period as a result of patient discomfort. The wearable device was removed prior to the completion of the monitoring period in two patients; one patient was discharged earlier than expected from the ICU, while another developed a diffuse drugassociated rash. Excluding patients whose devices were removed early, the devices were unable to detect a heart rate reading $4 \%$ of the time.

\section{Tachycardia and bradycardia detection}

We identified 13 SPO2-R-confirmed readings of bradycardia among four patients, all of whom were in sinus rhythm. Further statistical analysis was not done due to this small sample. The wearable had a sensitivity of $69.5 \%$ and specificity of $98.8 \%$ for the detection of tachycardia (Table 2 and Fig. 2). Among patients not in sinus rhythm $(n=8)$, the specificity for detecting tachycardia was similar (99.5\%), although sensitivity was worse (51.6\%). For faster heart rates (> $150 \mathrm{bpm})$, wearable device concordance with SPO2-R was poor. However, in many such cases, the wearable device reading showed better agreement with the true heart rate measured by continuous ECG, than did the SPO2-R readings, which tended to be falsely high.

\section{Sleep data}

A summary of the sleep quality data collected by the wearables is shown in Table 3. Among the 47 participants who had complete wearable sleep data recorded, the median wearable-reported sleep duration was $6.6 \mathrm{~h}$ (interquartile range [IQR] $2.7-13.5 \mathrm{~h}$ ) and the median number of sleep periods was 2 (IQR 1-4). Five participants $(11 \%)$ had no wearable device documented sleep for the entirety of the 24-h monitoring period. Among the 43 participants for whom the RCSQ was completed, the median total score was

Table 2 Test performance characteristics for personal fitness tracker detection of tachycardia, as compared to SPO2-R

\begin{tabular}{lll}
\hline & Sinus rhythm & Atrial fibrillation \\
\hline Sensitivity & 0.695 & 0.516 \\
Specificity & 0.988 & 0.995 \\
Positive predictive value & 0.948 & 0.983 \\
Negative predictive value & 0.914 & 0.804 \\
Accuracy & 0.92 & 0.836 \\
\hline
\end{tabular}

5.7/10.0 (IQR 2.7-8.0/10.0). There was a moderate correlation between wearable-derived sleep duration and total RCSQ score $(r=0.33, P=0.03,95 \%$ confidence interval $[\mathrm{CI}]$ 0.04, 0.58) (Fig. 3). The correlation between the percentage of nighttime asleep, as reported by the wearable device, and total RCSQ score was $0.36(P=0.02,95 \%$ CI $0.07,0.60)$. The correlation between the Fitbit-reported number of sleep periods and RCSQ-reported awakenings was $0.38 \quad(P=0.01$, $95 \%$ CI $0.09,0.61)$. There were no significant differences in wearable-reported sleep parameters between the CAM-ICU positive $(n=8)$ and CAM-ICU negative participants; however, $25 \%$ of CAM-ICU positive participants recorded no sleep over the entire 24-h monitoring period, compared to $8 \%$ of CAM-ICU negative participants.

\section{Device reusability}

Wearables were not found to be a significant source of pathogenic bacteria. Microbiologic sampling revealed bacteria consistent with commensal skin flora (Staphylococcus epidermidis) and/or environmental organisms (Bacillus species). S. epidermidis was only observed in samples taken prior to hydrogen peroxide disinfection, while Bacillus species were found in both pre- and post-disinfection specimens. Individual wearable devices were used between 5 and 13 times. There were no differences in wearable-SPO2-R heart rate correlations between the first and second half of the study $(P=0.18)$.

\section{Discussion}

The long-term adverse consequences of critical illness are increasingly being recognized as a research priority in critical care [19]. A growing body of research is now examining the determinants and potential modifiers of post-ICU recovery, including at least one study that made use of a wearable device to track patient movement and activity [20]. However, post-ICU recovery research currently lacks the richness of data available to researchers focused on the ICU stay itself since postdischarge data collection is limited to infrequent visits to follow-up clinics, or in many cases is nonexistent. New strategies are needed to collect data-ideally on a continuous basis-that better describes ICU recovery on the wards and in the patient's home environment.

To this end, we undertook an observational study to determine the feasibility of using a commercial-grade wearable device to monitor recovery after critical illness. Overall, the device was well tolerated and captured the vast majority of available data. For the detection of tachycardia, we found the wearable delivered high specificity and positive predictive value, but only low to moderate sensitivity. Much of the undercounting of fast heart rates 


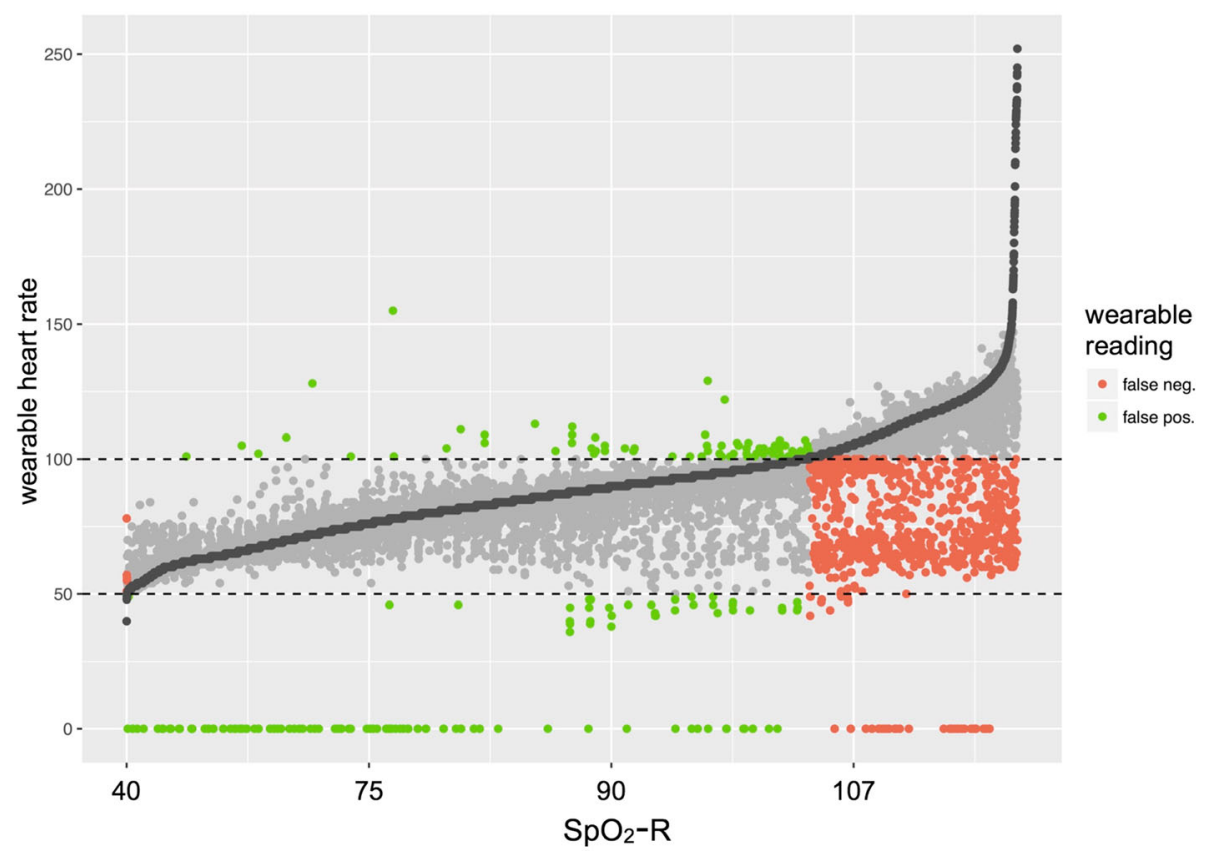

Fig. 2 Accuracy of wearable-derived heart rates for the detection of tachycardia $(H R>100)$ or bradycardia $(H R<50)$ as determined by SPO2 heart rates. The SPO2-derived values (dark gray) are shown sorted from lowest to highest heart rate. The corresponding wearable-derived heart rate is shown in either light gray (correct classification), green (false positive), or red (false negative). The majority of misclassified heart rates are false negatives for the detection of tachycardia. Some misclassification is due to wearable device readings of "0," reflecting data not captured by the device

by the wearable device was seen in patients who were not in sinus rhythm during at least some portion of the monitoring period. Compared to a validated sleep questionnaire, the wearable device had a moderate correlation with several metrics of sleep quality. Device performance

Table 3 Summary of wearable-reported and RCSQ sleep parameters

\begin{tabular}{lll}
\hline & Median & $(\mathrm{IQR})$ \\
\hline Wearable & & \\
Total sleep duration, hours & 6.6 & $(2.7-13.5)$ \\
Asleep time, hours & 6.1 & $(2.6-12.5)$ \\
Restless count & 7 & $(2.5-19.0)$ \\
Sleep quality A & 45.8 & $(38.0-63.5)$ \\
\# Sleep periods & 2 & $(1.0-4.0)$ \\
22:00-6:00 sleep as \% of total & $50 \%$ & $(15-80 \%)$ \\
\% of 22:00-6:00 asleep & $48 \%$ & $(3-84 \%)$ \\
RCSQ & & \\
Mean score & 5.7 & $(2.7-8.0)$ \\
1. Sleep depth & 5 & $(3.2-7.6)$ \\
2. Sleep latency & 6.2 & $(2.7-8.9)$ \\
3. Awakening & 5 & $(2.6-8.6)$ \\
4. Returning to sleep & 6.4 & $(2.1-9.1)$ \\
5. Sleep quality & 5.7 & $(1.6-8.6)$ \\
\hline
\end{tabular}

RCSQ Richards-Campbell Sleep Questionnaire did not appear to degrade over time. The wearables studied did not appear to be a significant source of nosocomial pathogens, although the presence of Bacillus species even after device cleaning suggests that spore-forming organisms could persist on some devices. Whether or not wearables would have to be reused at all would depend on their costs-which currently are relatively low-compared to the potential cost savings achieved with better clinical outcomes. The use of wearables to monitor convalescence after ICU discharge will ultimately pertain to patients who no longer require the resources of heavily monitored settings. To that end, our results are generalizable to a large contingent of patients, including post-ICU patients cared for on the wards, as well as those who have been discharged home.

In addition to their potential use following an ICU admission, wearables may also play a role in monitoring inpatients for signs of clinical deterioration, so as to identify as soon as possible any patient needing a higher level of care. Early Warning Systems (EWS) have been developed to address a "failure to rescue" problem, in which critical illness is identified too late [21]. Wearable devices stand to enhance data collection and monitoring both prior to and following an ICU admission, and as such is of growing importance in critical care research.

Interest in the clinical use of wearable devices and mobile health technology is increasing [2, 22]. While clinical evaluations of this technology remain scarce, some 


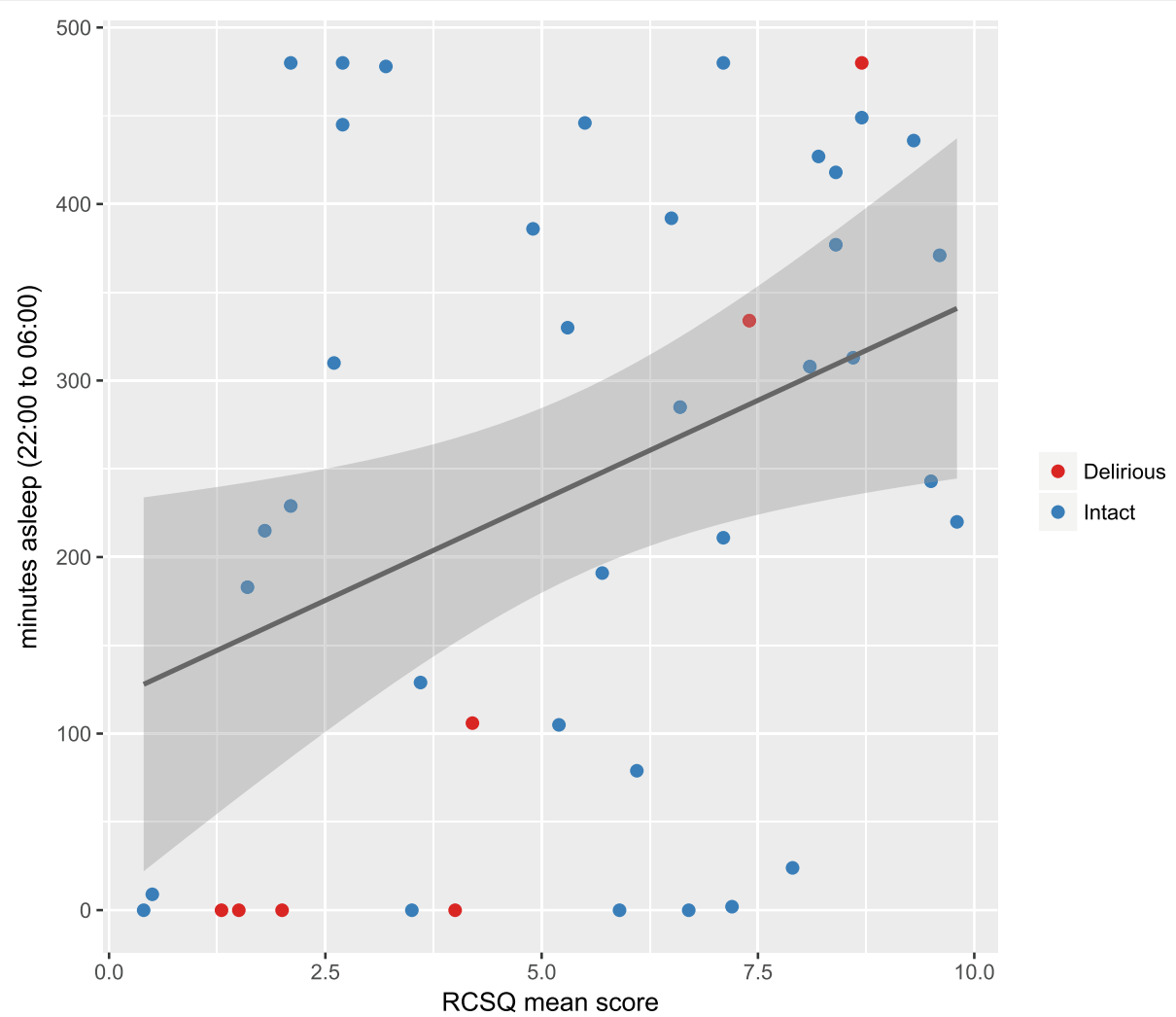

Fig. 3 Correlation between mean score on the Richards-Campbell Sleep Questionnaire (RCSQ) and wearable-derived measure of the number of minutes asleep overnight (between $22: 00$ and 06:00). The Pearson correlation coefficient was 0.33 ( $95 \% \mathrm{Cl} 0.04-0.58$ )

rigorous evaluations have been reported among healthy volunteers [4, 5] and among outpatients [23]. To our knowledge, this is the first study to examine the feasibility of using commercially available wearable devices among hospital inpatients to evaluate for heart rate derangements and sleep quality.

Wearables have the potential to become a useful tool in the early detection of critical illness. Heart rate is factored into the majority of EWS algorithms [24-28], and while the role of an EWS in reducing mortality remains unclear, there is evidence to suggest that these systems may be helpful [24]. Changes in heart rate may also portend changes in clinical status among ICU survivors on the wards or following hospital discharge. In this study, the high specificity but low to moderate sensitivity identified for the detection of tachycardia suggests that as currently configured, wearable-derived heart rate tracking would be highly specific, thereby mitigating alarm fatigue, but may lack sensitivity in some situations, resulting in missed detection of heart rate excursions. Ultimately, further confirmatory studies are required, which should also investigate alternate approaches to event detection, such as those based on proportional changes in heart rate. One potential limitation of wearable-enabled heart rate monitoring is a direct result of the PPG-based sensing mechanism employed, which may perform poorly in patients with a pulse deficit, such as those in atrial fibrillation.

Hospitalized patients often have a severely disrupted sleep, which may impair recovery [12]. Illness, medications, around-the-clock care activities, and environmental light and noise may contribute to perturbed sleep. Consumer-grade wearables with sleep monitoring capabilities could facilitate the routine evaluation of sleep among inpatients and the assessment of sleep-promoting interventions. Resource-intensive polysomnography (PSG) is impractical for routine sleep monitoring, and compliance with sleep questionnaires and sleep diaries is poor among inpatients [29]. Continuous data collection from wearables is passive and unobtrusive, and wearables are far less expensive than both PSG equipment and standard actigraphy devices.

Two recent studies have compared commercial-grade wearables with PSG in healthy subjects $[10,11]$. Mantua et al. found a strong correlation in total sleep time between wearable-derived data and PSG, and De Zambotti et al. found good agreement between wearables and PSG in measuring sleep, despite slight but significant overestimation of total sleep by the wearable devices. Altered sleep and activity patterns among inpatients may decrease the 
accuracy of wearables, which rely on movement to determine wakefulness, and could overestimate sleep in inpatients, who may be awake but immobile for long periods. The wearable device used in our study only counts periods of inactivity that exceed one hour as sleep, and may not capture fragmented naps, which are common in critically ill patients [30, 31].

Our study has a number of limitations that should be considered in interpreting the results. Conclusions regarding the influence of non-sinus rhythm on the accuracy of heart rate monitoring are limited by the relatively low prevalence of this condition in the study cohort, as are the findings relating sleep with delirium, which also had a low prevalence. While we considered the absence of sleep quality measures reported to indicate an absence of sleep during the monitoring period, an alternate interpretation is that these conditions reflect a failure of data capture. It is worth noting, however, that for the cases included that recorded no sleep data, heart rate data was successfully collected, making a failure of data capture an unlikely explanation for these findings. Lastly, differences between the internal clocks of the wearables and bedside monitors may have resulted in asynchronous heart rate recordings being treated as simultaneous, although correction factors were used in the analysis, and the time differences observed were shorter than the 5 min sampling interval of the wearable device.

\section{Conclusions}

In this observational study, we compared heart rate and sleep data recorded from a commercial-grade wearable device, with data from cardiac telemetry and sleep questionnaires. Devices showed high specificity and moderate sensitivity for the detection of tachycardia, with better performance in patients in sinus rhythm. Sleep quality metrics were moderately correlated with questionnaire data.

Future research in this area should focus on improving tachycardia detection, evaluating patients on the wards and at home, integrating wearable-derived data into the study of ICU recovery, and determining the impact of integrating wearable devices into hospital-wide EWS or rapid response services. Patients with arrhythmias should be studied as a subgroup in order to better define the accuracy of wearable-based heart rate sensing in this population. Further validation of sleep quality accuracy using other comparators such as PSG or conventional actigraphy would be useful, as would assessments of the accuracy of activity tracking.

\section{Additional file}

Additional file 1: Details regarding microbiologic assessment of the wearable devices as well as specifics regarding wearble device data capture and analysis. (PDF $49 \mathrm{~kb}$ )

\section{Abbreviations}

CAM: Confusion Assessment Method; ECG: Electrocardiography; EWS: Early

Warning System; HR: Heart rate; ICU: Intensive care unit; MRSA: Methicillin-resistant Staphylococcus aureus; PPG: Photoplethysmography; PSG: Polysomnography; RCSQ: Richards-Campbell Sleep Questionnaire; SPO2-R: Pulse oximetry pulse rate recordings (SPO2-R)

\section{Acknowledgements}

The authors wish to thank Miranda Hunt, Ilinca Georgescu, Tracy Boyd, and the nursing staff in the ICU at Kingston General Hospital.

\section{Funding}

This work was supported by an Innovation Fund award from the Southeastern Ontario Academic Medical Organization (SEAMO). Drs. Maslove and Boyd are supported by New Clinician Scientists awards from SEAMO.

\section{Availability of data and materials}

All primary data are available upon written request. Requests are subject to ethics approval and data sharing agreements.

\section{Authors' contributions}

RRK participated in primary data collection and analysis, data interpretation, and contributed to the drafting of the manuscript. EDM contributed to the analysis and interpretation of data and drafting of the manuscript. JGB contributed to the study concept and design, data analysis and interpretation, and revising of the manuscript. PS conducted the microbiologic sampling sub-study and contributed to the interpretation of results. DH contributed to the study concept and interpretation of results. MDW contributed to data collection and analysis and interpretation of results. DMM contributed to the study concept and design, data collection, data analysis, interpretation of results, and drafting of the manuscript. All authors read and approved the final manuscript.

Ethics approval and consent to participate

All participating patients, or substitute decision makers on their behalf, provided written informed consent for participation in this study. The Health Sciences Research Ethics Board at Queen's University reviewed and approved the study protocol, and the trial was registered with clinicaltrials.gov (NCT02527408).

\section{Consent for publication}

The patient depicted in Fig. 1 provided consent for the photo to be published in this manuscript.

\section{Competing interests}

The authors declare that they have no competing interests.

\section{Publisher's Note}

Springer Nature remains neutral with regard to jurisdictional claims in published maps and institutional affiliations.

\section{Author details}

${ }^{1}$ Department of Critical Care Medicine, Queen's University and Kingston Health Sciences Centre, Kingston, Ontario, Canada. ${ }^{2}$ School of Medicine, Queen's University, Kingston, Ontario, Canada. ${ }^{3}$ Department of Medicine, Queen's University and Kingston Health Sciences Centre, Kingston, Ontario, Canada. ${ }^{4}$ Department of Pathology and Molecular Medicine, Queen's University and Health Sciences Centre, Kingston, Ontario, Canada. ${ }^{5}$ Department of Emergency Medicine, Queen's University and Kingston Health Sciences Centre, Kingston, Ontario, Canada. ${ }^{6}$ Department of Neuroscience, Queen's University, Kingston, Ontario, Canada. ${ }^{7}$ Kingston Health Sciences Centre, Kingston General Hospital, Davies 2, 76 Stuart St., Kingston, Ontario K7L 2V7, Canada.

Received: 19 September 2017 Accepted: 8 November 2017 Published online: 21 November 2017

\section{References}

1. Salah H, Maclntosh E, Rajakulendran N. Wearable tech: leveraging canadian innovation to improve health: MaRS Market Insights; 2014 Mar 26. p. 1-45. 
Available from https://www.marsdd.com/wp-content/uploads/2015/02/ MaRSReport-WearableTech.pdf

2. Savage N. Mobile data: made to measure. Nature. 2015;527:S12-3.

3. El-Amrawy F, Nounou MI. Are currently available wearable devices for activity tracking and heart rate monitoring accurate, precise, and medically beneficial? Healthc Inform Res. 2015;21(4):315-6.

4. Case MA, Burwick HA, Volpp KG, Patel MS. Accuracy of smartphone applications and wearable devices for tracking physical activity data. JAMA-J Am Med Assoc. 2015;313(6):625-6.

5. Wang R, Blackburn G, Desai M, Phelan D, Gillinov L, Houghtaling P, et al. Accuracy of wrist-worn heart rate monitors. JAMA Cardiol. 2017;2(1):104-6.

6. Asada HH, Shaltis P, Reisner A, Rhee S. Mobile monitoring with wearable photoplethysmographic biosensors. IEEE Eng Med Biol Mag. 2003;22(3):28-40.

7. Tamura T, Maeda Y, Sekine M, Yoshida M. Wearable photoplethysmographic sensors_-past and present. Electronics. 2014;3(2):282-302.

8. Pantelopoulos A, Bourbakis NG. A survey on wearable sensor-based systems for health monitoring and prognosis. IEEE Trans Syst, Man, Cybern C. 2009; 40(1):1-12.

9. Di Rienzo M, Rizzo F, Parati G, Brambilla G, Ferratini M, Castiglioni P. MaglC system: a new textile-based wearable device for biological signal monitoring. Applicability in daily life and clinical setting. In: Engineering in medicine and biology society, 2005. IEEE-EMBS 2005. 27th annual international conference of the 2005 (pp. 7167-7169). Shanghai: IEEE.

10. de Zambotti M, Baker FC, Willoughby AR, Godino JG, Wing D, Patrick K, et al. Measures of sleep and cardiac functioning during sleep using a multisensory commercially-available wristband in adolescents. Physiol Behav. 2016;158:143-9.

11. Mantua J, Gravel N, Spencer R. Reliability of sleep measures from four personal health monitoring devices compared to research-based actigraphy and polysomnography. Sensors. 2016;16(5):646.

12. Young JS, Bourgeois JA, Hilty DM, Hardin KA. Sleep in hospitalized medical patients, part 1: factors affecting sleep. J Hosp Med. 2008;3(6):473-82.

13. Kamdar BB, Needham DM, Collop NA. Sleep deprivation in critical illness: its role in physical and psychological recovery. J Intensive Care Med. 2012; 27(2):97-111.

14. Litton E, Carnegie V, Elliott R, Webb SAR. The efficacy of earplugs as a sleep hygiene strategy for reducing delirium in the ICU: a systematic review and meta-analysis. Crit Care Med. 2016;44(5):992-9.

15. Richards KC, O'Sullivan PS, Phillips RL. Measurement of sleep in critically ill patients. J Nurs Meas. 2000;8(2):131-44.

16. Kamdar BB, Shah PA, King LM, Kho ME, Zhou X, Colantuoni E, et al. Patientnurse interrater reliability and agreement of the Richards-Campbell Sleep Questionnaire. Am J Crit Care. 2012;21(4):261-9.

17. Flannery $A H$, Oyler DR, Weinhouse GL. The impact of interventions to improve sleep on delirium in the ICU: a systematic review and research framework. Crit Care Med. 2016;44(12):2231-40.

18. Kroll RR, Boyd JG, Maslove DM. Accuracy of a wrist-worn wearable device for monitoring heart rates in hospital inpatients: a prospective observational study. J Med Internet Res. 2016;18(9):e253.

19. Herridge MS, Chu LM, Matte A, Tomlinson G, Chan L, Thomas C, et al. The RECOVER program: disability risk groups and 1-year outcome after 7 or more days of mechanical ventilation. Am J Crit Care Med. 2016;194(7):831-44.

20. McNelly AS, Rawal J, Shrikrishna D, Hopkinson NS, Moxham J, Harridge SD, et al. An exploratory study of long-term outcome measures in critical illness survivors: construct validity of physical activity, frailty, and health-related quality of life measures. Crit Care Med. 2016;44(6):e362-9.

21. Roney JK, Whitley BE, Maples JC, Futrell LS, Stunkard KA, Long JD. Modified early warning scoring (MEWS): evaluating the evidence for tool inclusion of sepsis screening criteria and impact on mortality and failure to rescue. J Clin Nurs. 2015;24(23-24):3343-54.

22. Semple JL, Armstrong KA. Mobile applications for postoperative monitoring after discharge. Can Med Assoc J. 2016. electronically published ahead of print; https://doi.org/10.1503/cmaj.160195.

23. Jakicic JM, Davis KK, Rogers RJ, King WC, Marcus MD, Helsel D, et al. Effect of wearable technology combined with a lifestyle intervention on long-term weight loss: the IDEA randomized clinical trial. JAMA. 2016;316(11):1161-71.

24. Smith MEB, Chiovaro JC, O'Neil M, Kansagara D, Quiñones AR, Freeman M, et al. Early warning system scores for clinical deterioration in hospitalized patients: a systematic review. Ann Am Thorac Soc. 2014;11(9):1454-65.
25. Churpek MM, Yuen TC, Park SY, Meltzer DO, Hall JB, Edelson DP. Derivation of a cardiac arrest prediction model using ward vital signs. Crit Care Med. 2012;40(7):2102-8

26. Churpek MM, Yuen TC, Winslow C, Meltzer DO, Kattan MW, Edelson DP. Multicenter comparison of machine learning methods and conventional regression for predicting clinical deterioration on the wards. Crit Care Med. 2016;44(2):368-74.

27. Churpek MM, Yuen TC, Park SY, Gibbons R, Edelson DP. Using electronic health record data to develop and validate a prediction model for adverse outcomes in the wards. Crit Care Med. 2014;42(4):841.

28. Churpek MM, Yuen TC, Winslow C, Hall J, Edelson DP. Differences in vital signs between elderly and nonelderly patients prior to ward cardiac arrest. Crit Care Med. 2015;43(4):816-22.

29. Bano M, Chiaromanni F, Corrias M, Turco M, De Rui M, Amodio P, et al. The influence of environmental factors on sleep quality in hospitalized medical patients. Front Neurol. 2014;5(4):267.

30. Fitbit Inc. Fitbit help: how do I track my sleep? [internet]. help.fitbit.com. [accessed 2016 Oct 15]. Available from: https://help.fitbit.com/articles/en_ US/Help_article/1314

31. Elliott R, McKinley S, Cistulli P, Fien M. Characterisation of sleep in intensive care using 24-hour polysomnography: an observational study. Crit Care. 2013;17(2):R46.

\section{Submit your next manuscript to BioMed Central and we will help you at every step:}

- We accept pre-submission inquiries

- Our selector tool helps you to find the most relevant journal

- We provide round the clock customer support

- Convenient online submission

- Thorough peer review

- Inclusion in PubMed and all major indexing services

- Maximum visibility for your research

Submit your manuscript at www.biomedcentral.com/submit
Biomed Central 